\title{
Intelligent Autonomous Vehicle for Indoor Mail Delivery Using Wireless Sensor Network
}

\author{
Mohammed Najm Abdullah ${ }^{1}$, Mohanad J. Ahmed ${ }^{2}$ \\ ${ }^{1,2}$ Department of Computer Engineering, University of Technology, Iraq \\ mustafamna@yahoo.com ${ }^{1}$,120567@student.uotechnology.edu.iq $q^{2}$
}

\begin{abstract}
The number of office transactions continually increasing ,therefore, several techniques proposed to improve quality of service for the office work. Improvements including electronic archiving limited; they didn't solve the problem of transferring parcels. Thus, paper transactions still exist inside a single office building and the transfer needed within a time limit. This leads to suggesting the autonomous vehicle for solving the delivery problem. In-order-to enable such the navigation within the indoor environment, an acceptable localization accuracy must exist. The wireless fingerprinting with Generalized Regression Neural Network (GRNN) for classification suggested in this research for localization estimation. Furthermore, the estimated location fused with the Odometer to bring more stable results. The Accuracy gained about $4.1 \mathrm{~cm}$ which enables the vehicle to localize and navigate. ZigBee modules with Arduino microcontrollers are the basic items of the research.
\end{abstract}

Index Terms-, GRNN, Fingerprinting, Odometry, ZigBee, MATLAB, Fusion.

\section{INTRODUCTION}

The object of localization is a large topic. Many researchers developed methods to get localization accuracy. The main topic in this field is the Global Positioning System (GPS) [1]. The GPS cannot run within the indoor environment because it needs at least three lineof-sight positioning satellites. Therefore, new methods were suggested for indoor localization to solve many of the problems in daily life. One problem is the everyday transactions (store and recover) documents growing up and became time-consuming, because of heavy traffic during a working day. Most offices adopted electronic archiving for speeding up the work.The offices still need paper, the electronic archiving reduced the search time and the store and recover only and didn't solve the way of transporting paper parcels in real-time [2]. The transported paper need to be localized, therefore it requires accuracy of the indoor environment. This research suggested the use of simple hardware such as ZigBee modules, enabling an autonomous vehicle to navigate for delivery targeted for this research. The rest of the paper is: first, a theoretical background then proposes system design discussed, after that implementation and results, then conclusions and future work.

\section{RELATED WORKS}

Different methods are used for delivery services and some of them have been quoted. Indoor localization can be defined as any system that can provide the location of objects in a closed structure such as hotels or rooms where the work is carried out for a long time without the presence of developers and depending on technologies such as odometer and 
laser ranging devices [5]. Many researchers studied the subject of autonomous services and sometimes called them robots suggested the Simultaneous Localization and Mapping (SLAM) for service robot [3]. The localization algorithms also optimized to bring more accuracy using wireless systems such as Zigbee modules and Particle Swarm Optimization (PSO) with GRNN [4]. The use of odometer is important to some applications and fuzed to increase the accuracy of the localization systems[2]. While there are studies proposed the navigation without a predefined map by taking a random paths for long period of times to cover areas such as the dust cleaner robot supplied with infrared proximity sensors that can move freely within indoor environment assuming the random movements will cover all the target area with time [6]. Other studies solved the problem of re-localization when the service robot lost by using the wireless fingerprinting algorithms [10]. Also, the intelligent mail delivery robot adopted in [11] which meets the objectives of the assumed office environment.

\section{THEORETICAL BACKGROUND}

\section{A. FINGERPRINTING LOCALIZATION}

This algorithm used to compare pre-measured known locations received signal strength indicator (RSSI) values from the offline mode. The RSSI values stored in a database with its $\mathrm{x}$, and $\mathrm{y}$ coordinates for later comparison in the online mode. Equation 1 where $\mathrm{n}$ is the path loss exponent and A is the measured RSSI reference for 1 meter, and $\mathrm{d}$ is the distance in meter. The value earned from the formula is in $(-\mathrm{dB})$. There are many references in the literature explaining the procedure of fingerprinting localization in a wireless sensor network such as [7].

$$
\operatorname{RSSI}_{j}(d)=-\left(10 \times n \times \log _{10}(d)\right)+A
$$

\section{B. ZIGBEE}

ZigBee is a wireless network protocol IEEE 802. 15. 4-based designed to be used with low-datarate sensor and control networks. ZigBee is the standard for Wireless Sensor Networks (WSNs), targeted for low battery, low-cost applications, the localization using fingerprinting done by measuring the RSSI from ZigBee modules. The choice of where to place ZigBee units can affect the localization accuracy and subject to trial and error. The fingerprinting method does not need to know the anchor's position, the only major concern is getting signal strength measurements and building radio map. For further information about ZigBee technology refer to [8].

\section{GENERALIZED REGRESSION NEURAL NETWORK (GRNN)}

This algorithm provides a smooth transition from one viewed value to another and can be used for any regression problem in which an assumption of linearity is not justified such as the correlated RSSI readings from beacons. There are many types of neural networks used for different purposes according to their design such as Probabilistic Neural Networks (PNN) for classification problems. The GRNN is used for function approximation and regression. The fingerprinting lookup classified as function approximation and has the advantage of learning in one pass through data and generalized from examples as soon as they stored and converge to the conditional mean regression surface as more examples viewed, and gets away from poor solutions exist in the local minima of error (compared to the iterative neural networks methods). The training input and target data restored from the offline fingerprinting, real-time input data and then predict its estimated location. The 
neural network trained and getting the values of the BASE (for each $\mathrm{x}$ and $\mathrm{y}$ coordinates of the mobile beacon separately to increase the accuracy of regression results) by considering the neural network to be unconstrained nonlinear minimization problem targeted to minimum mean square Euclidian distance between the real and estimated location. MATLAB optimization toolbox functions used for this purpose[4].

\section{WHEELED LOCOMOTION MODEL}

The two-wheel differential-drive is the most popular design and composed of two active fixed wheels equipped with stepper motors placed at a fixed distance parallel to each other. Every stepper motor controlled separately to bring flexibility in motion. The turning is a rotation about the center of the line between the two wheels marked by the (+) sign (Figure 1). The wheels placed in the center of the chassis, caster wheels placed in the front and rear those special passive wheels to ensure the vehicle's balance and they have no mathematical effect [9].

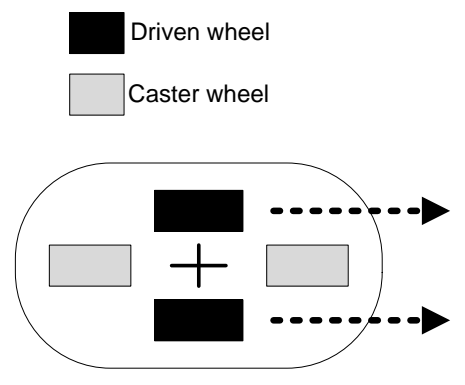

(a)

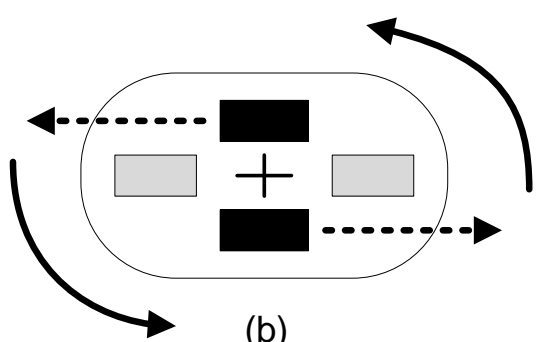

(b)

FigURE 1 DRIVING AND TURNING DIFFERENTIAL DRIVE (TOP VIEW)

The equal speed for both motors is forwarding movement. Different speeds will rotate the vehicle around its center in a zero turning radius (Figure 1-b).

The planned navigation depending on the accuracy of localization, each moment the location was estimated the vehicle ordered to move a step forward, once the source and destination verified a trip started.

By setting different wheel direction and angle of the controlled vehicle. To estimate the distance the following method stated in [9] is used.

$\begin{array}{ll}r & \text { wheel radius } \\ d & \text { Distance between driven wheels } \\ \text { tick_per_rev } & \text { Number of encoder ticks for one full wheel revolution } \\ \text { ticks_l } & \text { Number of ticks during measurements in the left encoder } \\ \text { ticks_r } & \text { Number of ticks during measurements in right encoder } \\ s_{L} & \text { Distance of left wheel } \\ s_{R} & \text { Distance of right wheel }\end{array}$

Dividing the measured ticks by the number of ticks per revolution gives the number of wheel revolutions and multiplying this by the wheel circumference gives the traveled distance in meters [9].

$$
\begin{aligned}
& s_{L}=2 \pi r \frac{\text { ticks_l }}{\text { ticks_per_rev }} \\
& s_{R}=2 \pi r \frac{\text { ticks_r }}{\text { ticks_per_rev }}
\end{aligned}
$$




$$
s=\frac{s_{L}+s_{R}}{2}
$$

The vehicle will receive a command either to move forward according to Figure 1.

This formula works for driving forward or turning on the spot according to the following.

If $s_{L}=s_{R}, s_{L}>0$, driving strightforward.

If $s_{L}>s_{R}$ e.g. $s_{L}=2 \times s_{R}$, driving in a right curve.

$\dot{\theta_{R}}, \dot{\theta_{L}}$ are individual wheel speeds in revolutions per second

$$
\begin{aligned}
& v_{R}=2 \pi r \cdot \dot{\theta_{R}} \\
& v_{L}=2 \pi r \cdot \dot{\theta_{L}}
\end{aligned}
$$

$w$ Is the rotation speed

$v$ Is the linear speed

Thus the required wheel speeds for the desired motion by finding the inverse kinematics

$$
\left[\begin{array}{c}
\dot{\theta_{L}} \\
\dot{\theta_{R}}
\end{array}\right]=\frac{1}{2 \pi r}\left[\begin{array}{cc}
1 & -\frac{d}{2} \\
1 & \frac{d}{2}
\end{array}\right]\left[\begin{array}{c}
v \\
w
\end{array}\right]
$$

\section{E. SENSOR FUSION}

The weighted average method is a direct technique for data fusion. This paper assumes each data source got has equal weight for simplicity of calculations according to the following formulas [2].

dist_odo is the distance estimated from the odometry encoder.

dist_finger is the distance estimated from fingerprinting localization.

$$
\text { Fusion }=(\text { dist_odo }+ \text { dist_finger }) / 2
$$

\section{PROPOSED SYSTEM DESIGN}

The proposed system contains a localization algorithm developed using wireless fingerprinting as stated in 3.1 along with the GRNN for classification stated in 3.3. The second part of the system is the autonomous vehicle which uses the localization technique. According to section 3.4,

The proposed vehicle $(25 \mathrm{~cm} \times 15 \mathrm{~cm} \times 8 \mathrm{~cm})$ has two active wheels placed at the center of chassis for not taking too much area while turning in narrow places such as office corridor. The position of active wheels requires two additional passive wheels placed at the front and rear to ensure stability. The ZigBee components are used to enable communication between the central computer and the microcontroller to receive control commands and also deliver odometry readings from the vehicle to the central computer. The passive wheels neglected in mathematical calculations so they not shown in the architecture Figure 2. 


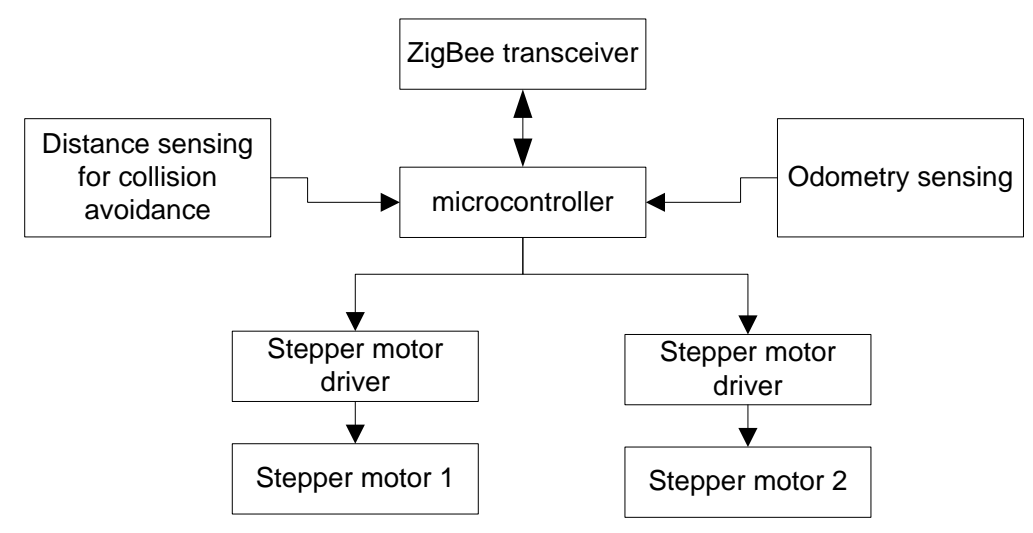

Figure 2 Autonomous VEHICLE ARCHITECTURE

The vehicle equipped with two stepper motors driven by h-bridge drivers pulsed from the microcontroller. With the h-bridge drivers, the speed and direction of each active wheel can be controlled to give three types of motion (forward, left, and right), the backward not included in this research. The ultrasonic sensors for wall alignment, they can also tell if the vehicle in-front-of a door by measuring the total distance from both sides.

The overall system integrated using dedicated software functions set developed using MATLAB platform, while Figure 3 shows the hardware architecture of autonomous delivery.

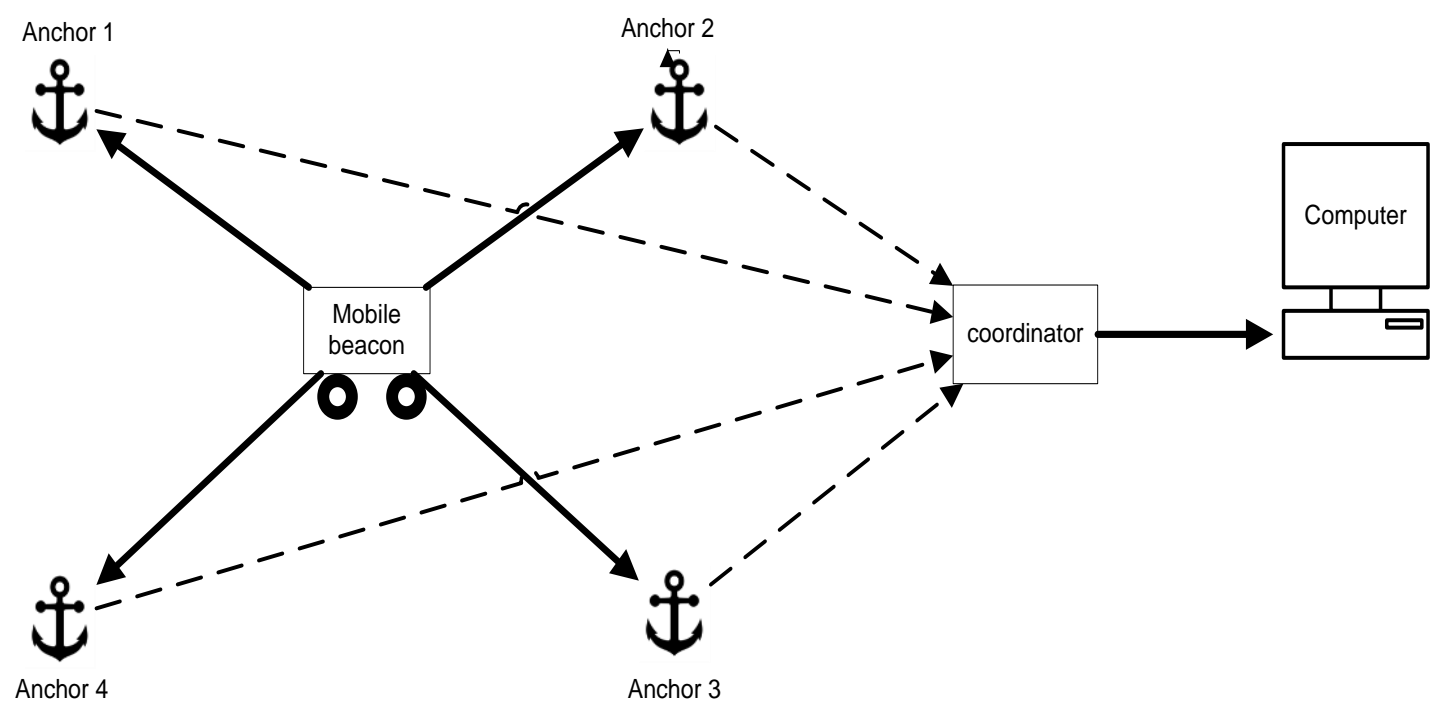

FIGURE 3 HARDWARE ARCHITECTURE OF AUTONOMOUS MAIL DELIVERY SYSTEM

The beacon used by fingerprinting localization attached to vehicle and system became mobilebeacon fixed anchor. Following are steps of operation:

- Mobile beacon broadcasts a beacon frame to anchors.

- Upon receiving a beacon frame by anchors they activating their event-driven procedure and measure the signal strength of the received frame.

- Each anchor knows its time slice to take its turn sending signal strength readings to the sink in time slicing manner to avoid collision Figure 4.

- The computer collecting readings, as well as odometer feedback, then estimate the current position. 
- Upon estimating the current position, the computer software will command the vehicle either to stop if it reaches the destination or gives a command to move to the next position toward the destination.

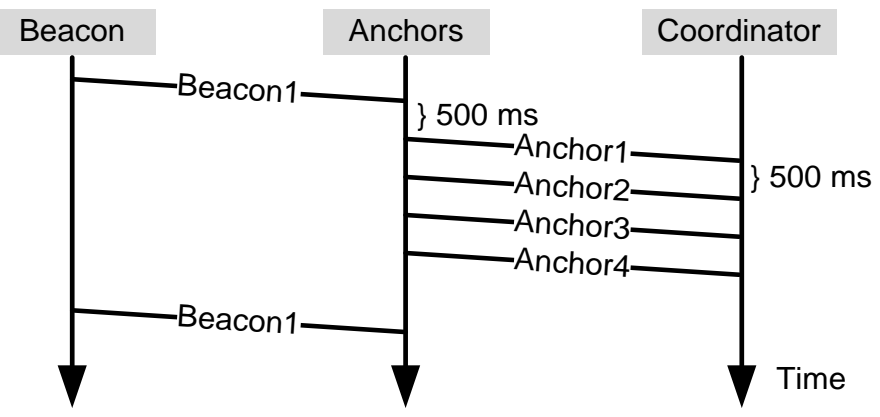

FigURE 4 TIMING EVENTS INTERACTION AMONG SENSOR NODES

\section{IMPLEMENTATION AND RESULTS}

The vehicle is set-up in place about $4 \times 4 \mathrm{~m}$ and four anchors spread in the corners of the area as stated in Figure 4. The recorded points as the vehicle move the real points recorded shown in Table 1 which also shows readings got from GRNN location estimation and fusion with vehicle's odometry.

TABLE 1OBTAINED RESUltS

\begin{tabular}{cccccccc}
\hline \multicolumn{2}{c}{ real coordinates } & \multicolumn{2}{c}{ estimated coordinates GRNN } & \multicolumn{2}{c}{ Odometry } & \multicolumn{2}{c}{ fusion } \\
$\mathrm{x}(\mathrm{m})$ & $\mathrm{y}(\mathrm{m})$ & $\mathrm{x}(\mathrm{m})$ & $\mathrm{y}(\mathrm{m})$ & $\mathrm{x}(\mathrm{m})$ & $\mathrm{y}(\mathrm{m})$ & $\mathrm{x}(\mathrm{m})$ & $\mathrm{y}(\mathrm{m})$ \\
\hline 0.15 & 1 & 0.15 & 1.05 & 0.15 & 1 & 0.44507 & 1.478692 \\
0.3 & 1 & 0.3109 & 1.05 & 0.3 & 1 & 0.738172 & 1.11061 \\
0.45 & 1 & 0.4263 & 1.0568 & 0.45 & 1 & 0.519795 & 1.423322 \\
0.6 & 1 & 0.6 & 1.05 & 0.6 & 1 & 1.098627 & 1.432156 \\
0.75 & 1 & 0.75 & 1.05 & 0.75 & 1 & 0.974507 & 1.471221 \\
0.9 & 1 & 0.9761 & 1.0668 & 0.9 & 1 & 0.998852 & 1.281378 \\
1.05 & 1 & 1.0496 & 1.05 & 1.05 & 1 & 1.285104 & 1.169287 \\
1.2 & 1 & 1.1984 & 1.05 & 1.2 & 1 & 1.557781 & 1.264437 \\
1.35 & 1 & 1.35 & 1.05 & 1.35 & 1 & 1.511468 & 1.127176 \\
1.5 & 1 & 1.5092 & 1.0505 & 1.5 & 1 & 1.520942 & 1.0444 \\
1.65 & 1 & 1.5464 & 1.0721 & 1.65 & 1 & 1.935102 & 1.513456 \\
1.8 & 1 & 1.8 & 1.05 & 1.8 & 1 & 2.261217 & 1.170052 \\
1.95 & 1 & 1.9494 & 1.05 & 1.95 & 1 & 2.006038 & 1.040696 \\
2.1 & 1 & 2.1001 & 1.05 & 2.1 & 1 & 2.342838 & 1.2981 \\
2.25 & 1 & 2.1964 & 0.9648 & 2.25 & 1 & 2.25561 & 0.990358 \\
2.4 & 1 & 2.3999 & 1.05 & 2.4 & 1 & 2.890746 & 1.12449 \\
2.55 & 1 & 2.55 & 1.05 & 2.55 & 1 & 2.836266 & 1.027382 \\
2.7 & 1 & 2.7 & 1.05 & 2.7 & 1 & 2.727383 & 1.355288 \\
2.85 & 1 & 2.3369 & 0.779 & 2.85 & 1 & 2.809697 & 1.304606 \\
3 & 1 & 2.9897 & 1.0497 & 3 & 1 & 3.122427 & 1.134218 \\
\hline & & & & & & & \\
\hline
\end{tabular}


The difference in Eucledian distance between estimated and real points, average error earned from GRNN about $8.3 \mathrm{~cm}$ and reduced to $4.1 \mathrm{~cm}$ after fusing the odometry compared to real points of trajectory (Figure 5).

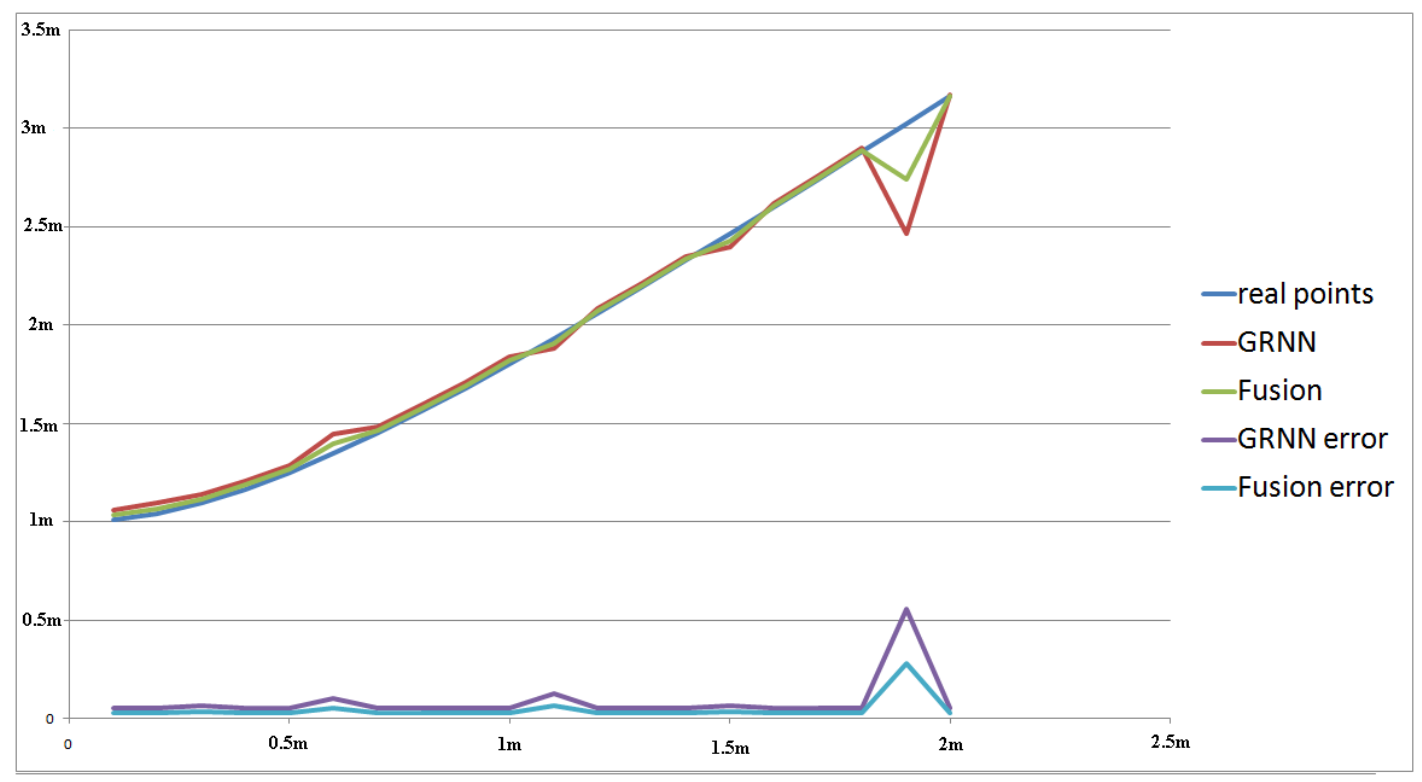

Figure 5 EUCLIDIAN DISTANCE (M) BETWEEN REAL AND ESTIMATED POINTS

It is worth to say that the stepper motor brought accuracy for estimating odometry in this case and error which was read from odometry alone was $1 \mathrm{~cm}$ and neglected in calculations.

\section{DISCUSSION}

The purpose of this study is to enable autonomous transport based on indoor localization technology. Getting accuracy is a key in this study. Results show improvement in localization accuracy about $4.1 \mathrm{~cm}$ while fusing the Odometer with the averaging operation as the simplest type of fusing data. With this accuracy got the vehicle able to navigate and deliver parcels. While sudden variation in the results due to environmental change such as opening and closing a door. Using stepper motor support results by getting accurate step estimation and lessen sudden changes by using odometer. The use of additional fusion algorithms enhanced results.

For long-distance navigation, the measurements of odometer accumulate errors in position estimation because of manufacturing errors of stepper motors, although they are almost identical, in addition, there are errors of slipping tires, also the difference in diameter of the tires. Therefore vehicle will run on short steps to reduce accumulative errors and fuse these estimations with fingerprinting localization estimation which represents a global localization within the indoor environment which do not contain cumulative errors, thus taking advantage of both features to produce reliable results.

Autonomous indoor delivery is a contribution to offices that suffer from large traffic. The stepper motor gives accuracy more improvement and stability by estimating steps traveled this study limited to the indoor environment. To expand the study to an outdoor environment a hybrid technique used to travel between the indoor and outdoor environment. Autonomous delivery improves the quality of service of the office work and other similar services such as restaurants and hotels. 


\section{CONCLUSIONS AND SUGGESTIONS}

For localization algorithms used by GRNN, the average error is about $8 \mathrm{~cm}$. With the assist of odometer error reduced to $4.1 \mathrm{~cm}$. The effect of variation in signal strength readings decreased by using odometer. With this accuracy, the vehicle is able to navigate and the purpose of the proposed system was achieved using simple hardware such as ZigBee and Arduino. Further improvements to fusion function will increase accuracy. The measured signal strength if used alone is not reliable. The method need to perform outdoor to see how results are. For recommendations, the use of mechanum wheels brings more flexibility in motion while navigating indoor. The use of different technology such as ultra-wideband will enhance the accuracy of indoor localization because of its immunity to multipath effect.

\section{REFERENCES}

[1]S. Alraih, A. Alhammadi, I. Shayea and A. M. Al-Samman, "Improving accuracy in indoor localization system using fingerprinting technique," 2017 International Conference on Information and Communication Technology Convergence (ICTC), Jeju, 2017, pp. 274-277.

[2]Dobrev, Yassen, Peter Gulden, and Martin Vossiek. "An indoor positioning system based on wireless range and angle measurements assisted by multi-modal sensor fusion for service robot applications." IEEE Access 6 (2018): 69036-69052.

[3]J. Zhang, Y. Ou, G. Jiang and Y. Zhou, "An approach to restaurant service robot SLAM," 2016 IEEE International Conference on Robotics and Biomimetics (ROBIO), Qingdao, 2016, pp. 2122-2127.

[4]Zou, Ziming, Fumin Li, and Xinxin Qiao. "Research on Node Location Algorithm of ZigBee Based on PSOGRNN Neural Network." Proceedings of the International Symposium on Big Data and Artificial Intelligence. ACM, 2018.

[5] Pinillos, R., Marcos, S., Feliz, R., Zalama, E., \& Gómez-García-Bermejo, J. (2016). Long-term assessment of a service robot in a hotel environment. Robotics and Autonomous Systems, 79, 40-57.

[6] Saleem, Adeel, Atif Iqbal, and Adnan Sabir. "Design and Implementation of an Intelligent Dust Cleaner Robot for Uneven and Nonstructural Environment." 2019 2nd International Conference on Computing, Mathematics and Engineering Technologies (iCoMET). IEEE, 2019.

[7]Ahson, Syed A., and Mohammad Ilyas, eds. Location-based services handbook: Applications, technologies, and security. CRC Press, 2010.

[8]Farahani, Shahin. ZigBee wireless networks and transceivers. Newnes, 2011, pp. 235-242.

[9]Siciliano, Bruno, and Oussama Khatib, eds. Springer handbook of robotics. Springer, 2016, pp. 391-410.

[10] Luo, Ren C., and Tung Jung Hsiao. "Kidnapping and Re-Localizing Solutions for Autonomous Service Robotics." IECON 2018-44th Annual Conference of the IEEE Industrial Electronics Society. IEEE, 2018.

[11] Bhaskar, K., Mansooor, A., Anand, K. R., \& Ramani, A. (2011, September). Intelligent mail delivery robot. In 2011 2nd International Conference on Intelligent Agent \& Multi-Agent Systems (pp. 1-6). IEEE. 